\title{
Relação da Atividade Profissional com Desvios Posturais e Encurtamentos Musculares Adaptativos
}

\begin{abstract}
Guilherme Garcia Holderbaum* é graduado emEducação Física pela Universidade do Vale do Rio dos Sinos - UNISINOS e Especialista em MedicinaeCiências do Esporte, pelaPUC/RS - SOGAMED. Cláudia Tarragô Candotti ** é mestre em ciências do Movimento Humano, professora adjunta do curso de Educação Física da UNISINOSe doutoranda do Programa de Pós-Graduação em Ciências do Movimento Humano, da ESEF/UFRGS. Ana Maria Steffens Pressi *** é graduada emEducação Física pela Universidade do Vale do Rio dos Sinos - UNISINOS e mestranda do Programa de Pós-Graduação em Ciências do Movimento Humano, daESEF/UFRGS. Janete Christianus **** é graduada em
\end{abstract} Educação Física pela Universidade do Vale do Rio dos Sinos - UNISINOS.

\section{Resumo}

O objetivo deste estudo foi verificar se as más posturas adotadas no ambiente de trabalho podem favorecer o surgimento de desvios posturais. A amostra foi constituída por dezenove funcionários do departamento de limpeza da UNISINOS. Foram realizadas avaliações posturais e goniométricas para verificar, respectivamente, as alterações posturais e as amplitudes de movimento articular (ADM). Os resultados mostraram que $\mathbf{1 0 0 \%}$ dos funcionários apresentaram, pelo menos, um tipo de desvio postural e sugerem que o exercício da atividade profissional em posturas inadequadas favorece à instalação de desvios posturais. Ambas avaliações, posturais e goniométricas, quando correlacionadas, apresentaram diferenças significativas $(\mathbf{p} £ 0,05)$ na comparação das médias de ADM entre os funcionários com segmentos corporais dentro e fora do alinhamento postural normal.

Descritores: Atividade profissional; Desvios posturais; Movimento articular.

\section{Abstract \\ The purpose of this study was to verify if the bad adopted postures in the work atmosphere, during the accomplishment of professional activities can favor the appearance of posture deviations. The s ample was made}

up of 19 employees of the cleaning department from UNISINOS. The methodology used in this research was constituted by the accomplishment of posture goniometrical evaluarions in order to verify the posture alterations and the amplitude of articular movements, respectively. The obtained results showed that $100 \%$ of the individuals presented at least a type of posture deviation. These results suggest that professional acdvities when practised $n$ inadequate postures favor the installaüon of posture deviadons. The posture and goniometrical evaluations, when correlated presented significant dirferences $(p £ 0,05)$ in comparison with the averages of ADM among the individuais with corporal segments inside and out the norma lposture alignment.

Keywords: Work; Posture; Articular movement; Spine injured.

\section{Introdução}

A postura do homem sofreu várias alterações no decorrer da história e da evolução do ser humano, de modo que a coluna vertebral, em razão de sua constante posição vertical e das cargas que pressionam determinadas áreas, passou a apresentar doenças e degenerações. A coluna vertebral funciona como uma espécie de alicerce para que o restante do corpo possa se sustentar, sendo que esta sustentação depende de um equilíbrio muscular dinâmico (Candotti, 1993; Fischinger, 1984). Vários autores apresentam o mesmo ponto de vista, considerando a postura como

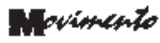

Movimento, Porto Alegre, V. 8, n. 1, p. $21-29$, janeiro/abril 2002 
o arranjo relativo das partes do corpo, a disposição das partes do corpo no espaço para a realização de uma atividade específica (Kendall, Mccreary \& Provance, 1995;Kisner\&Colby, 1992;Lehmkuhl \&Smith, 1989).

$\mathrm{O}$ advento da industrialização e a evolução dos meios de transporte e suporte para a vida em geral também favoreceram o surgimento das doenças relativas a coluna vertebral, sendo denominadas por alguns, como doenças da civilização moderna, que estão intimamente ligadas com as atividades da vida diária (AVD) e com o trabalho dos indivíduos. Segundo Rasch (1991), um encurtamento adaptativo de músculos pode ocorrer se as posturas habituais e um trabalho pesado crônico

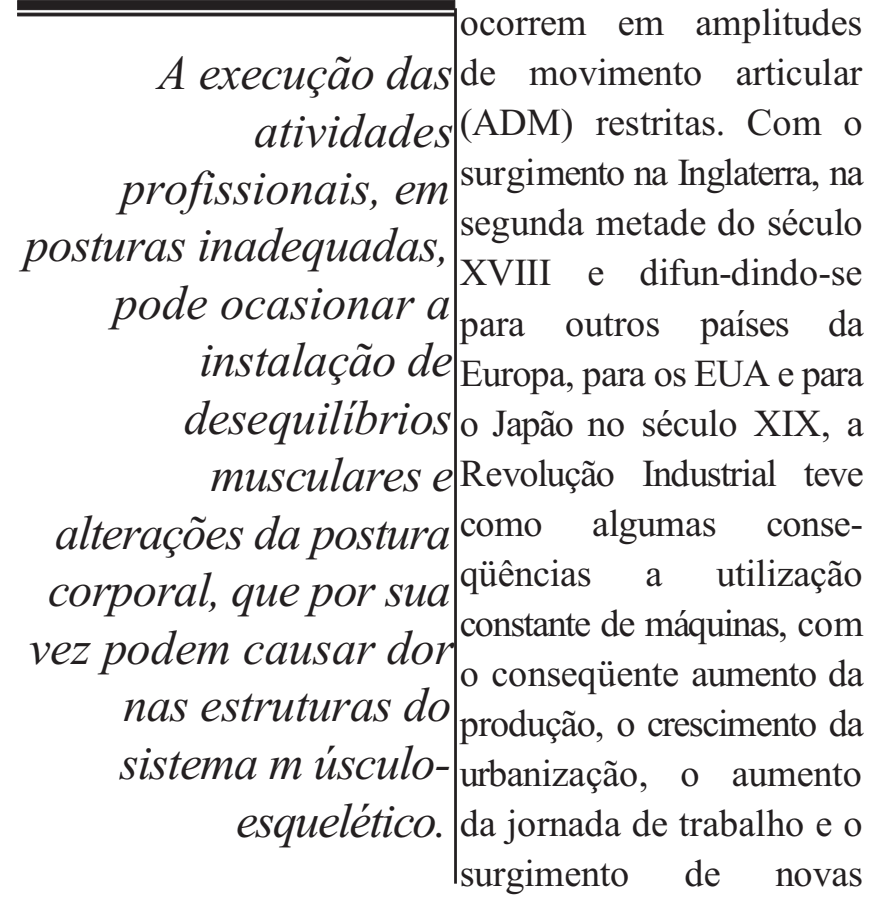
profissões (Piletti \& Piletti, 1995). Uma das profissões mais antigas é a atividade de limpeza que na época, consistia apenas em realizar as tarefas domésticas na própria residência. Hoje, essa profissão ganhou novos campos de trabalho, passando a ser realizada nas ruas pelas prefeituras e nas instituições de ensino, nos condomínios e em empresas de pequeno, médio e grande porte.

A atividade de limpeza é exercida por ambos os sexos, por pessoas das mais variadas idades e em todo e qualquer estabelecimento que se queira manter limpo e em ordem. Esta função exige dos indivíduos maior força e resistência musculares e conscientização da postura corporal, pois é uma profissão que baseia-se na execução de movimentos repetitivos e unilaterais, que associados a longos períodos de trabalho e somados a anos de profissão, pode favorecer à instalação de desvios posturais. Desse modo, passamos a considerar a necessidade de avaliar periodicamente estes indivíduos a fim de levantar subsídios para melhorar a qualidade de vida dos mesmos, uma vez que a detecção de desvios posturais, ainda no início de sua formação, torna possível a minimização dos efeitos que estes possam causar (Kendall, Mccreary \& Provance, 1995).

Para avaliar os indivíduos que desempenham a atividade de limpeza acreditamos que avaliações do tipo postural e goniométrica, sejam de grande importância, pois objetivam detectar os desvios posturais e os encurtamentos musculares adaptauvos, respectivamente. Compreendemos a importância destas avaliações, a partir do entendimento de que a execução das atividades profissionais, em posturas inadequadas, pode ocasionar a instalação de desequilíbrios musculares e alterações da postura corporal, que por sua vez podem causar dor nas estruturas do sistema músculo-esquelético.

A importância de manter o bem estar biopsicossocial da população, deve ser uma preocupação constante dos profissionais da área da saúde. Estes profissionais, atuando em equipes multidisciplinares, podem contribuir muito para a saúde do trabalhador. Atualmente, o profissional de Educação Física vem sendo considerado como uma importante "ferramenta" das empresas sensíveis as necessidades de mudanças de comportamento e valorização de seus recursos humanos. Este profissional passa a ser, então, um dos responsáveis pelo desenvolvimento de fórmulas que preservem e fortaleçam os níveis de saúde dos indivíduos das mais diversas áreas.

Os objetivos de nosso estudo foram: (1) verificar a incidência de desvios posturais em trabalhadores da

\section{2}


atividade de limpeza através de avaliações posturais e goniométricas e (2) verificar as correlações existentes entre os resultados das avaliações posturais e goniométricas.

Nossas hipóteses eram de que: (1) a postura adotada por trabalhadores do departamento de limpeza, no ambiente de trabalho, favoreceria a ocorrência de alterações posturais e (2) se as posturas adotadas, por trabalhadores do departamento de limpeza, no ambiente de trabalho, durante a realização de suas tarefas, tendessem a instalação de desequilíbrios musculares, então esta atividade profissional favoreceria a limitações da amplitude de movimento articular.

\section{Materiais e métodos}

\section{População e Amostra}

Esse estudo foi do tipo expôs facto, sendo a amostra intencional, constituída por dezenove trabalhadores do departamento de limpeza da UNISINOS, entre 23 e 48 anos de idade, de ambos os sexos. A média de tempo de serviço no setor foi de 3,97 anos e o desvio padrão foi de 2,45 . Todos os indivíduos trabalhavam, em média, quarenta 44 horas por semana, sendo que setenta porcento $(70 \%)$ possuíam o primeiro grau completo. Todos os indivíduos consentiram em participar do estudo.

\section{Procedimentos de Aquisição}

Foram realizadas duas avaliações: (1) avaliação postural estática e (2) avaliação goniométrica. Para a realização das avaliações, foi solicitado aos indivíduos que ficassem de pés descalços e apenas calção para os homens e bermuda e mini blusa para as mulheres, sendo avaliado um de cada vez.

\section{Avaliação Postural Estática}

Para a realização da avaliação postural estática foi utilizado um posturógrafo, que permitiu observações da postura corporal nas posições lateral e posterior. Os pontos de referência utilizados foram o maléolo lateral e o ponto central entre os calcanhares do indivíduo, nas posições lateral e posterior, respectivamente (Kendall,Mccreary\&Provance, 1995).

A observação na posição lateral permitiu constatar as alterações da postura do indivíduo no que se refere ao: (1) pescoço (normal, para frente, hiperestendido ou retraído), (2) ombros (normal, para frente ou rodado), (3) escapulas (normal ou abduzida), (4) coluna vertebral (hipercifose, hiperlordose ou retificação lombar), (5) abdômen (normal ouprotuso), (6) quadril (normal, antevertido ou retrovertido) e (7) joelho (normal, flexum ou recurvatum).

A observação na posição posterior permitiu constatar as alterações de postura no que se refere ao: (1) ombro (normal, assimétrico), (2) escapulas (normal, assimétrica), (3) coluna vertebral (se existe indicativos de presença de escoliose), (4) triângulo de Tales (simétrico ou assimétrico), (5) espinha ilíaca póstero superior - EIPS (normal, assimétrica) e (6) joelho (normal, valgo ou varum).

\section{Avaliação Goniométrica}

Para a realização da avaliação goniométrica foi utilizado um goniômetro. O goniômetro é um instrumento que serve para medir ângulos articulares e determinar a amplitude de movimento das articulações (Kendall, Mccreary \& Provance, 1995). Para este estudo foi necessário confeccionar um goniômetro e, para isso, foram utilizados: (1) um goniômetro pequeno, (2) uma régua de cinqüenta centímetros, que foi colada no goniômetro, para servir de haste e (3) dois níveis colados no goniômetro, um para leitura vertical e outro para leitura horizontal, que permitiram o alinhamento do instrumento com o segmento corporal no momento da avaliação (Figura 1).

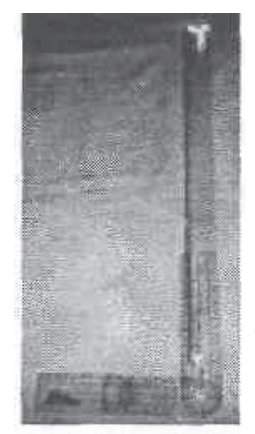

Figura 1 - Goniômetro confeccionado para este estudo, sendo que a seta destaca os níveis para leituras vertical e ho- 
A avaliação goniométrica foi realizada por dois avaliadores, um responsável pela leitura do goniômetro e outro responsável pela estabilização das articulações, sendo que a leitura do goniômetro foi expressa em graus. $\mathrm{O}$ avaliador responsável pela leitura colocava o goniômetro na posição recomendada de teste (Norkin \& White, 1997) e acompanhava o movimento da articulação até a amplitude máxima atingida pelo avaliado $\mathrm{e}$, ao final do movimento, realizava a leitura do instrumento, que era registrado em ficha individual. A estabilização foi utilizada durante as provas para conseguir uma melhor fixação, a fim de limitar a ação desejada a uma articulação específica. $\mathrm{O}$ avaliador responsável pela estabilização exerceu essa função de forma manual provocando uma força externa sobre o segmento corporal avaliado e cuidando para que, através da estabilização manual, não limitasse a amplitude de movimento articular do indivíduo avaliado.

Neste estudo foram avaliadas duas articulações: (1) articulação escápulo-umeral e (2) articulação coxofemural, sendo que nesta última também se avaliou a amplitude de movimento articular dos músculos biarticulares do joelho.

$\mathrm{Na}$ articulação escápulo-umeral, foram avaliados os movimentos de flexão, rotação interna e rotação externa do ombro, dos lados esquerdo e direito. $\mathrm{Na}$ articulação coxo-femural foram avaliados os movimentos de rotação interna e rotação externa do joelho e os músculos biarticulares do joelho (reto femural, isquiotibiais, sartório e tensor da fáscia-lata) de ambos os lados.

\section{Procedimentos de Análise}

\section{Avaliação Postural Estática}

No momento da avaliação, conforme a observação do avaliador, eram registrados em uma ficha padrão os desvios observados para cada indivíduo. Após, foi realizado uma discriminação por segmento corporal visando quantificar percentualmente cada desvio observado em cada segmento corporal.

\section{Avaliação Goniométrica}

Após o registro dos ângulos articulares em fichas individuais de avaliação, foi realizado um levantamento de todos os ângulos articulares medidos em cada um dos 16 testes das 2 articulações e dos músculos biarticulares do joelho, com 8 testes. Os dados foram então, posteriormente, submetidos ao tratamento estatístico, o que também possibilitou a correlação desta avaliação com a avaliação postural.

\section{Tratamento Estatístico}

O teste estatístico utilizado neste estudo foi o teste $\mathrm{t}$ simples (para amostras independentes) que possibilitou a comparação das médias das avaliações posturais dos indivíduos com postura corporal normal e com alterações da postura corporal e das avaliações goniométricas. O nível de significância adotado foi de 0,05 .

\section{Resultado e discussão}

As avaliações posturais e goniométricas foram aplicadas para detectar a incidência de desvios posturais em trabalhadores do departamento de limpeza da UNISINOS e verificar a presença de encurtamentos musculares.

Analisando as avaliações posturais estáticas, foi possível verificar algumas alterações de postura corporal, relevantes para este estudo. Na Figura 2 podemos observar a variação percentual da postura corporal dos funcionários do departamento de limpeza da UNISINOS, nas posições lateral e posterior, durante a avaliação postural estática.

As avaliações posturais, juntamente com as avaliações goniométricas, quando submetidas ao tratamento estatístico de comparações de médias mostraram que todas as médias de ADM da articulação escápuloumeral foram maiores para os indivíduos que apresentaram alterações da posição do ombro na postura corporal, quando avaliados com o posturógrafo, sendo que houve diferença significativa em dois testes, conforme pode ser observado na Tabela I.

Estes resultados sugerem que, os indivíduos que apresentaram a posição do ombro normal na avaliação 


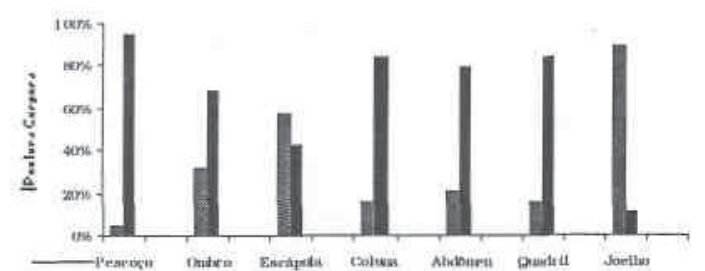

A

Normal EAlterado

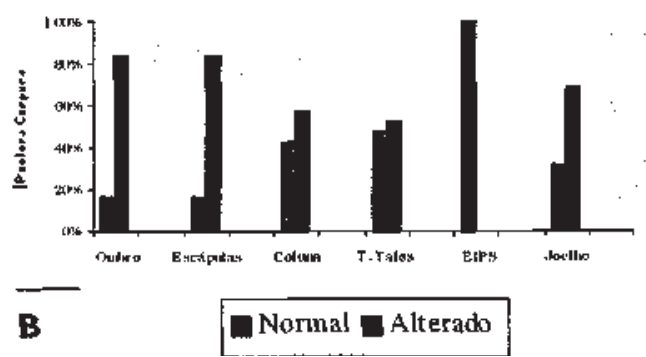

Figura 2 - Variação percentual da postura corporal dos trabalhadores do departamento de limpeza da UNISINOS, (A) na posição lateral e (B) na posição posterior, durante a avaliação postural estática.

\begin{tabular}{l|c|c|c|c}
\hline \multicolumn{1}{c|}{ Articulação escápulo-umeral } & Postura de Ombro & Média & Desvio Padrão & Valor $p^{*}$ \\
\hline lexão Direita & Normal & 120,33 & 5,43 & 0,219 \\
& Alterada & 125,54 & 9,19 & \\
\hline${ }^{\text {n} l e x a ̃ o ~ E s q u e r d a ~}$ & Normal & 116,83 & 4,62 & $0,008 * *$ \\
& Alterada & 128,23 & 11,75 & \\
\hline cotação Interna Direita & Normal & 57,33 & 15,89 & 0,268 \\
& Alterada & 65,53 & 5,62 & \\
\hline cotação Interna Esquerda & Normal & 56,16 & 13,99 & $0,009 * *$ \\
& Alterada & 69,38 & 6,07 & \\
\hline cotação Externa Direita & Normal & 67,00 & 14,72 & 0,322 \\
& Alterada & 72,53 & 9,01 & \\
\hline cotação Externa Esquerda & Normal & 69,83 & 16,26 & 0,802 \\
& Alterada & 71,30 & 9,24 & \\
\hline Valor $p$ referente ao teste t em eada uma das comparações * $p$ & \\
menor do que 0 nível de significâneia & $a<0,05$ &
\end{tabular}

Tabela I - Comparação das médias e desvios padrões dos testes de ADM da articulação Escápulo-Umeral com a postura do ombro normal e alterada, quando submetido ao teste $t$.

postural, possuem uma ADM menor, na articulação escápulo-umeral, do que os indivíduos que apresentam desvios e/ou alterações da posição do ombro na postura corporal.

Os resultados das médias de ADM da articulação escápulo-umeral, quando analisada a posição da escapula, não apresentaram diferença significativa. As médias de ADM foram próximas, tanto para os indivíduos que apresentaram a escapula normal como

\begin{tabular}{l|c|c|c|c}
\hline \multicolumn{1}{c|}{ Articulação escápulo-umeral } & Postura de Escapula & Média & Desvio Padrão & Valor p * \\
\hline Flexão Direita & Normal & 123,82 & 7,44 & 0,964 \\
& Alterada & 124,00 & 10,13 & \\
\hline Flexão Esquerda & Normal & 123,09 & 9,89 & 0,502 \\
& Alterada & 126,75 & 13,40 & \\
\hline Rotação Interna Direita & Normal & 64,09 & 10,33 & 0,586 \\
& Alterada & 61,37 & 10,79 & \\
\hline Rotação Interna Esquerda & Normal & 65,45 & 12,71 & 0,913 \\
& Alterada & 64,87 & 8,62 & \\
\hline Rotação Externa Direita & Normal & 69,72 & 13,20 & 0,636 \\
& Alterada & 72.25 & 7,66 & \\
\hline Rotação Externa Esquerda & Normal & 71,63 & 13,97 & 0,734 \\
& Alterada & 69,75 & 7,44 & \\
\hline * Valor $p$ referente ao teste tem cada uma das comparações \\
**p menor do que o nível de significâneia cr^0,05
\end{tabular}

Tabela II - Comparação das médias dos testes de ADM da articulação Escápulo-Umeral com a postura da escapula normal e alterada quando submetidas ao teste $\mathrm{t}$.

para aqueles que apresentaram alterações da posição da escapula na postura corporal, quando avaliados com o posturógrafo. Estes resultados podem ser observados na Tabela II e sugerem que tanto os indivíduos que apresentam a posição da escapula normal na avaliação postural, quanto os indivíduos que apresentam alterações da posição da escapula na postura corporal possuem médias de ADM aproximadas, não havendo assim, uma relação neste caso entre a postura corporal e a ADM.

Ao analisar os resultados da articulação coxo-femural, verificamos que não houve diferença significativa entre as médias de ADM desta articulação para os indivíduos que apresentam a posição do quadril normal $\mathrm{e}$ alterada, embora os valores tenham sido maiores para os indivíduos que apresentam a posição do quadril normal, quando avaliados com o posturógrafo, conforme pode ser observado na Tabela III.

\begin{tabular}{l|c|l|c|c}
\hline \multicolumn{1}{c|}{ Articulação Coxo-Femura! } & Postura do Quadril & Média & Desvio Padrão & Valor $p^{*}$ \\
\hline Rotação Interna Direita & Normal & 25,00 & 6,24 & 0,197 \\
& Alterada & 19,93 & 5,96 & \\
\hline Rotação Interna Esquerda & Normal & 25,00 & 4,58 & 0,140 \\
& Alterada & 20,37 & 4,77 & \\
\hline Rotação Externa Direita & Normal & 27,00 & 5,56 & 0,140 \\
& Alterada & 22,62 & 4,33 & 0,062 \\
\hline Rotação Externa Esquerda & Normal & 28,66 & 8,08 & \\
\hline & Alterada & 21,75 & 5,06 & \\
\hline * Valor $p$ referente ao leste t em eada uma das comparações ** \\
$p$ menor do que o nível de significâneia (Xá 0,05 \\
\hline
\end{tabular}

Tabela III - Comparação das médias dos testes de ADM da articulação Coxo-Femural com a postura do quadril normal e alterado quando submetido ao teste $t$. 
A especialização Os resultados dos testes dos ou o sedentarismo joelho demonstraram que de certas profissões, houve diferença con tribuem para. significativa em dois debilitar o testes, sendo que as médias funcionamento de ADM destes músculos fisiológico, para $\left.\right|_{\text {indivíduos }} ^{\text {foram maiores }}$ para os deformações apresentaram a posição do estruturais, epara quadril normal, quando aumentara avaliados com o probabilidade de posturógrafo. Estes doenças ocupacionais. resultados podem ser observados na Tabela IV e sugerem que, os indivíduos que apresentaram a posição do quadril normal na avaliação postural, possuem uma ADM maior, nos músculos biarticulares do joelho, do que os indivíduos que apresentam desvios e/ou alterações da posição do quadril na postura corporal.

\begin{tabular}{|c|c|c|c|c|}
\hline Músculos Biarticulares do Joelhe & Postira do Quadrial & Média & Desvio Padräo & Valor $p^{*}$ \\
\hline Reto Femural Dircio & \begin{tabular}{|c|} 
Normal \\
Altertula \\
\end{tabular} & $\begin{array}{l}38.33 \\
36,75 \\
\end{array}$ & $\begin{array}{l}9,29 \\
9,92\end{array}$ & $0.80 !$ \\
\hline Rew Femural Esquerdo & $\begin{array}{l}\text { Nomial } \\
\text { Allerada }\end{array}$ & $\begin{array}{l}40,100 \\
43,00 \\
33,00\end{array}$ & $\begin{array}{l}4,00 \\
8,82\end{array}$ & 0,203 \\
\hline Isquiocibiais Direito & $\begin{array}{l}\text { Nermal } \\
\text { Alterada }\end{array}$ & $\begin{array}{l}67,66 \\
52,00\end{array}$ & $\begin{array}{l}12,66 \\
11,47\end{array}$ & $0,047 * *$ \\
\hline Isquirtribiais Esquerdo & $\begin{array}{l}\text { Normal } \\
\text { Alletrada }\end{array}$ & $\begin{array}{l}58,66 \\
53,75 \\
\end{array}$ & $\begin{array}{l}10,50 \\
12,48 \\
1\end{array}$ & 0,533 \\
\hline Sartério Direito & $\begin{array}{l}\text { Nomal } \\
\text { Alterada }\end{array}$ & $\begin{array}{l}33,33 \\
21,43 \\
\end{array}$ & 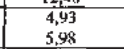 & $0,005 * *$ \\
\hline Sartório Esquerdo & $\begin{array}{l}\text { Normal } \\
\text { Alterivala }\end{array}$ & $\begin{array}{l}25,00 \\
22,62\end{array}$ & $\begin{array}{l}5,00 \\
5,40\end{array}$ & 0,491 \\
\hline Tetasour da Fúscia Lata Dircittr & $\begin{array}{l}\text { Normal } \\
\text { Alteradal } \\
\end{array}$ & $\begin{array}{l}22,00 \\
22,81\end{array}$ & $\begin{array}{l}3,46 \\
4,26\end{array}$ & 0.761 \\
\hline 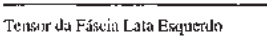 & $\begin{array}{l}\text { Normal } \\
\text { Alterálata }\end{array}$ & \begin{tabular}{|l|}
24,66 \\
20,18 \\
\end{tabular} & $\begin{array}{l}5,03 \\
4,96\end{array}$ & 0,171 \\
\hline
\end{tabular}

Tabela IV - Comparação das médias dos testes de ADM dos Músculos Biarticulares do Joelho com a postura de quadril normal e alterada quando submetidos ao teste $\mathrm{t}$.

Os trabalhadores do departamento de limpeza da UNISINOS apresentaram desvios posturais tanto na vista lateral quanto na vista posterior da avaliação postural, bem como variações de ADM nos testes de goniometria. Ao buscarmos uma correlação entre as avaliações, postural e goniométrica, verificamos que houve algumas diferenças significativas entre as com- parações das médias das duas avaliações.

A avaliação goniométrica da flexão esquerda e rotação interna esquerda da articulação escápulo-umeral indicou que as médias dos testes de ADM dos indivíduos que apresentaram alterações da posição do ombro, quando avaliados com o posturógrafo, são maiores que do que as médias dos testes de ADM dos indivíduos que apresentaram o ombro na posição normal. $\mathrm{O}$ procedimento estatístico indicou que as médias dos testes de ADM apresentaram diferenças significativas entre os indivíduos com alterações e com ombro na posição normal.

$\mathrm{Na}$ comparação das médias dos testes de $\mathrm{ADM}$ da articulação coxo-femural na avaliação goniométrica, entre os indivíduos que apresentaram a posição do quadril normal ou alterada, quando avaliados com o posturógrafo, verificou-se que os indivíduos com a posição do quadril normal apresentaram médias de ADM maiores do que os indivíduos que apresentaram a posição do quadril alterada, entretanto, não houve diferença significativa entre as médias de ADM. Contudo, pode-se afirmar que, não existe relação entre as médias de ADM da articulação coxo-femural com a posição do quadril normal ou alterada na avaliação postural.

Na comparação das médias das ADM dos testes dos músculos biarticulares (isquiotibial direito e sartório direito) do joelho, na avaliação goniométrica, entre os indivíduos que apresentaram a posição do quadril normal ou alterada, quando avaliados com o posturógrafo, constatamos que houve diferença significativa entre as médias, pois os indivíduos com alterações da posição do quadril possuem uma ADM maior do que os indivíduos com a posição do quadril normal. A ADM normal varia entre os indivíduos, sendo influenciada por fatores como a idade, o sexo, a ausência de movimentos e hábitos posturais inadequados (Norkin \& White, 1997). Os hábitos posturais causados por fatores ambientais, provocam desequilíbrios musculares, acarretando assim, em limitações da ADM (Kendall, Mccreary \& Provance, 1995; Kisner\&Colby, 1992). 
Os fatores biomecânicos são apontados como os mais diretamente relacionados ao disparo inicial das doenças ocupacionais, embora os fatores pessoais, psicossociais e organizacionais possam agravar ou perpetuar o quadro clínico instalado. A repetição, as posturas inadequadas, as grandes amplitudes de movimento e o uso de força excessiva são considerados os principais causadores das doenças ocupacionais (Putz, 1988). As doenças ocupacionais são decorrentes da combinação de vários fatores a que o trabalhador está exposto durante a execução de sua atividade profissional (Sjogarrd \& Sogaard, 1998). Em um estudo realizado por Lio, Coury \& Oishi (1999) para identificar os fatores de risco biomecânicos em três diferentes setores produtivos (manual, semi-automatizado e automatizado) de uma empresa, foi verificado que as atividades profissionais realizadas em níveis de automatização podem ser mais prejudiciais à saúde dos trabalhadores, que aquelas executadas manualmente. $\mathrm{O}$ ritmo da tarefa passa a ser ditado pela máquina, obrigando o sujeito a adotar posturas inadequadas e realizar movimentos com grande freqüência para acompanhar a velocidade da máquina.

Desse modo, acreditamos que o trabalho, quando executado sob determinadas condições, pode causar danos irreparáveis à saúde da coluna vertebral do indivíduo. $\mathrm{Na}$ atualidade, existe uma multidão de pessoas jovens com problemas de dores na coluna vertebral porque, além de posturas inadequadas no ambiente de trabalho e nas AVDs, associa-se também a tensão psíquica a que normalmente as pessoas jovens são submetidas, devendo lutar para vencer na vida, ganhar dinheiro, comprar carro, casa e aproveitar os anos, além da tensão da existência na cidade grande, com medo de assaltos, com a preocupação do trânsito, etc (Knoplich,1986).

Notamos que há uma unanimidade entre os autores quando afirmam que os problemas posturais são desenvolvidos pelos indivíduos no ambiente de trabalho, quando exercem suas funções em condições ambientais precárias, posturas inadequadas ou executando os mesmos movimentos, várias horas por dia, durante anos. Do mesmo modo, verificamos uma unanimidade entre os autores quando defendem a idéia de que o trabalho, função, ocupação, atividade profissional ou mesmo AVDs, causam danos a coluna vertebral, quando executados em posturas desfavoráveis.

Desta forma, inferimos que os nossos resultados sinalizam que os desvios posturais encontrados estão associados à postura adotada durante a realização da atividade. Portanto, aceitamos a hipótese (1) deste estudo, pois a atividade profissional, quando exercida, supostamente, em posturas inadequadas, favoreceu o surgimento dos desvios posturais, bem como dos desequilíbrios musculares encontrados nos trabalhadores do departamento de limpeza da UNISINOS.E, rejeitamos a hipótese (2), em função dos desvios pos-Embora muitos turais e dos desequilíbrios estudos têm musculares provocarem limitações identificado daADM, ao contrário do movimentos que abordado ante- repetitivos, forças riormente e referenciado excessivas e posturas na literatura. extremas como fatores Os efeitos da tecnologia, de risco para as lesões bem como das mudanças músculo-esqueléticas, na organização do são escassos os estudos trabalho sobre os que avaliam trabalhadores, suas quantitativamente condições de vida, de estes riscos em relacionamento e saúde, vêm sendo estudados e situações pesquisados há algum|ocupacionais reais tempo (Cañete, 1996). Nas grandes cidades e na população brasileira em geral, a partir da década de sessenta, quando se deu início a civilização do automóvel, o indivíduo passou a fazer tudo, ou quase tudo, sem se locomover, sem fazer exercícios, e além do mais, trabalhando com instrumentos ou operando máquinas e assumindo posturas inadequadas durante todo o expediente (Knoplich, 1986). Muitas vezes o indivíduo tem que

\section{Mrimento}


trabalhar em condições desfavoráveis e em ritmo excessivamente intenso, em posições fadigantes e deformadoras, antinaturais ou violentas. A especialização ou o sedentarismo de certas profissões, contribuem para debilitar o funcionamento fisiológico, para deformações estruturais, e para aumentar a probabilidade de doenças ocupacionais (Oliveira, 1990).

Cailliet (1979) ao por em evidência a enorme parcela da população que sofre de dor nas costas, afirma que cerca de $80 \%$ da população mundial civilizada, apresentam problemas de dores relacionados à coluna vertebral. Isso ocorre porque as pessoas, de um modo geral, passaram a trabalhar em máquinas, sentadas em posições incorretas, levantando peso de maneira inadequada, realizando movimentos causadores de alta tensão na estrutura da coluna vertebral ou produzindo torções no tronco ou na cabeça durante o expediente de trabalho muitas horas, durante muitas semanas, meses e anos consecutivos. As inúmeras profissões que surgiram depois da Revolução Industrial, no fim do século passado, têm produzido um maior número de portadores de problemas na coluna vertebral.

A eliminação de movimentos e posturas críticas durante a atuação profissional ou durante as atividades da vida diária são fatores relevantes na redução da incidência de problemas relativos a coluna vertebral (Couto, 1998). Rasch (1977) salienta que os hábitos defeituosos de postura são causados tanto por traumatismos e doenças, como por fatores ambientais e ocupacionais. Couto (1998) afirma que para reduzir a incidência de desvios posturais relacionados ao ambiente de trabalho, é necessário reduzir a tensão excessiva em articulações e tendões bem como, que a execução de atividades profissionais seja em posturas adequadas. Embora muitos estudos têm identificado movimentos repetitivos, forças excessivas e posturas extremas como fatores de risco para as lesões músculo-esqueléticas, são escassos os estudos que avaliam quantitativamente estes riscos em situações ocupacionais reais (Snook, 1995). Portanto acreditamos que estudos como este, que buscam avaliar desvios posturais, se fazem necessários, principalmente se associados com fatores ergonômicos, para que se possa promover alterações dos fatores causais dos desvios posturais e melhorar a qualidade de vida dos trabalhadores, em geral.

\section{Conclusão}

Os principais resultados deste estudo sugerem que a atividade profissional, quando supostamente executada em posturas inadequadas, aliadas a movimentos repetitivos e unilaterais, durante um período de tempo prolongado, favoreceu o surgimento de desvios posturais e desequilíbrios musculares nos trabalhadores do departamento de limpeza da UNISINOS.

Acreditamos ser importante dar continuidade a este estudo, e para tanto, aumentar o grupo de amostragem, a fim de obter resultados mais consistentes de modo à posteriormente, aplicá-los a outras profissões para a realização de uma possível relação entre desvios posturais em diferentes tipos de profissão. 


\section{Referências bibliográficas}

CAILLIET, Rene. Lombalgias. $1^{\circ}$ edição, São Paulo, Ed. Manole, 1979.

CANDOTTI, Cláudia Tarragô. $\mathrm{O}$ papel da Educação Física na formação de bons hábitos posturais nas crianças em idade escolar. Educação para crescer, p. 18-20; Porto Alegre, 1993.

CANETE, Ingrid. Humanização: Desafio da empresa moderna: A ginástica laborai como um caminho. Porto Alegre, Ed. Artes e Ofícios, 1996.

COUTO, Hudson de Araújo. Como gerenciar a questão das L.E.R.. D.O.R.T.: lesões por esforços repetitivos e distúrbios osteomusculares relacionados ao trabalho. $1^{\circ}$ edição, Belo Horizonte, Ed. Ergo, 1998.

FISCHINGER, Bárbara Sybille. A escoliose vista por um fisioterapêuta: uma visão didática. $\mathrm{I}^{\mathrm{O}}$ edição, Caxias do Sul, Ed. Educs, 1982.

, Bárbara Sybille. Escoliose em fisioterapia. ${ }^{1 o}$ edição, São Paulo, Ed. Panamed, 1984.

JACOB, Stanley W; FRANCONE, Clarice Ashworth; LOSSOW, Walter J. Anatomia e Fisiologia Humana. $5^{\circ}$ edição, Rio de Janeiro, Ed. Guanabara Koogan, 1990.

KENDALL, Florence P; MCCREARY, Elizabeth K; PROVANCE, Patrícia G. Músculos: Provas e Funções. $4^{\circ}$ edição, São Paulo, Ed. Manole, 1995-

KISNER, Carolyn; COLBY, Lynn A. Exercícios Terapêuticos: Fundamentos e Técnicas. $2^{\circ}$ edição, São Paulo, Ed. Manole, 1992.

KNOPLICH, J. Enfermidades da Coluna Vertebral, S.P.: Panamed Editorial, 1986.

LÉO, J. A., COURY, H. J. C. \& OISHI, J. Identificação de Fatores de Risco Biomecânicos em Atividades Ocupacionais Manuais e Automatizadas. VIII Congresso Brasileiro de Biomecânica, 1999.

LEHMKUHL, L. Don; SMITH, Laura K. Brunnstrom: Cinesiologia Clínica. $4^{\circ}$ edição, São Paulo, Ed. Manole, 1989.

NORKIN, Cynthia C; WHITE, D. Joyce. Medida do movimento Articular: Manual da Goniometria. $2^{0}$ edição, Porto Alegre, Ed. Artes Médicas, 1997.

OLIVEIRA, Chrysóstomo Rocha. Manual Prático de L.E.R. $2^{\text {a }}$ edição, Belo Horizonte, Ed. Health, 1998.

PILETTI, Nelson; PILETTI, Claudino. História e Vida: Da idade moderna à atualidade. $14^{\circ}$ edição, São Paulo, Ed. Ática S.A., 1995-

PUTZ, Anderson. Cumulative Trauma Disorders: a Manual for Musculoskeletal diseases of the upper limb. London. Taylor and Francis, 1988.

RASCH, Philip J. Cinesiologia e Anatomia aplicada a Ciência do movimento Humano. $\mathrm{I}^{\mathrm{O}}$ edição, Rio de Janeiro, Ed. Guanabara Dois, 1977.

, Philip J. Cinesiologia e Anatomia Humana. $7^{\circ}$ edição, Rio de Janeiro, Ed. Guanabara Koogan, 1991-

SJOGAARD, G., SOGAARD, K. Muscle Iniurv in Repetitive Motion Disorders. Clinical Orthopedics and Related Research, $\mathrm{n}^{\circ} 351$, $\mathrm{p}$. 21-31, 1998.

SNOOK, S. H. et all. Psychophvsical Studies of Repetitive Wrist Flexion and Extension. Ergonomics, 38 (7), p. 1488-1507, 1995.

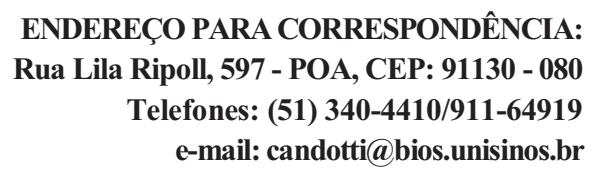

Recebido em: 16.04.01 Revisado em: 01.03 .02 Aceito em: 20.03 .02 
